\title{
Cerebrospinal fluid cytokines in multiple system atrophy: A cross-sectional Catalan MSA registry study
}

\author{
Yaroslau Compta $^{\mathrm{a}, *, 1}$, Sara P. Dias ${ }^{\mathrm{a}, \mathrm{b}, 1}$, Darly M. Giraldo ${ }^{\mathrm{a}}$, Alexandra Pérez-Soriano ${ }^{\mathrm{a}}$, \\ Esteban Muñoz ${ }^{\mathrm{a}}$, Josep Saura ${ }^{\mathrm{c}}$, Manel Fernández ${ }^{\mathrm{a}}$, Paloma Bravo ${ }^{\mathrm{a}}$, Ana Cámara ${ }^{\mathrm{a}}$, \\ Marta Pulido-Salgado ${ }^{\mathrm{a}}$, Cèlia Painous ${ }^{\mathrm{a}}$, José Ríos ${ }^{\mathrm{d}}$, María José Martí ${ }^{\mathrm{a}, * *}$, on behalf of the CMSAR \\ consortium (Javier Pagonabarraga ${ }^{e}$, Francesc Valldeoriola ${ }^{a}$, Jorge Hernández-Vara ${ }^{f}$, \\ Serge Jauma Classen ${ }^{g}$, Victor Puente ${ }^{\mathrm{h}}$, Claustre Pont ${ }^{\mathrm{i}},{\text { Núria Caballol, }{ }^{\mathrm{j}} \text { Eduardo Tolosa }}^{\mathrm{a}}$, \\ Angels Bayes ${ }^{\mathrm{k}}$, Jaume Campdelacreu ${ }^{\mathrm{g}}$, Oriol de Fàbregues ${ }^{\mathrm{f}}$, Asunción Ávila ${ }^{\mathrm{j}}$, Matilde Calopa ${ }^{\mathrm{g}}$, \\ Carles Gaig ${ }^{\mathrm{a}}$, Neus Fabregat ${ }^{\mathrm{l}}$, Pau Pastor ${ }^{\mathrm{m}}$, Miquel Aguilar ${ }^{\mathrm{m}}$, Montserrat Pujol $^{\mathrm{n}}$, \\ Almudena Sánchez ${ }^{\mathrm{a}}$, Lluís Planellas ${ }^{\mathrm{a}}$, Mario Ezquerra ${ }^{\circ}$, Rubén Fernández-Santiago ${ }^{\circ}$, \\ Teresa Botta ${ }^{\mathrm{P}}$, Gian Tartaglia ${ }^{\mathrm{P}}$ )
}

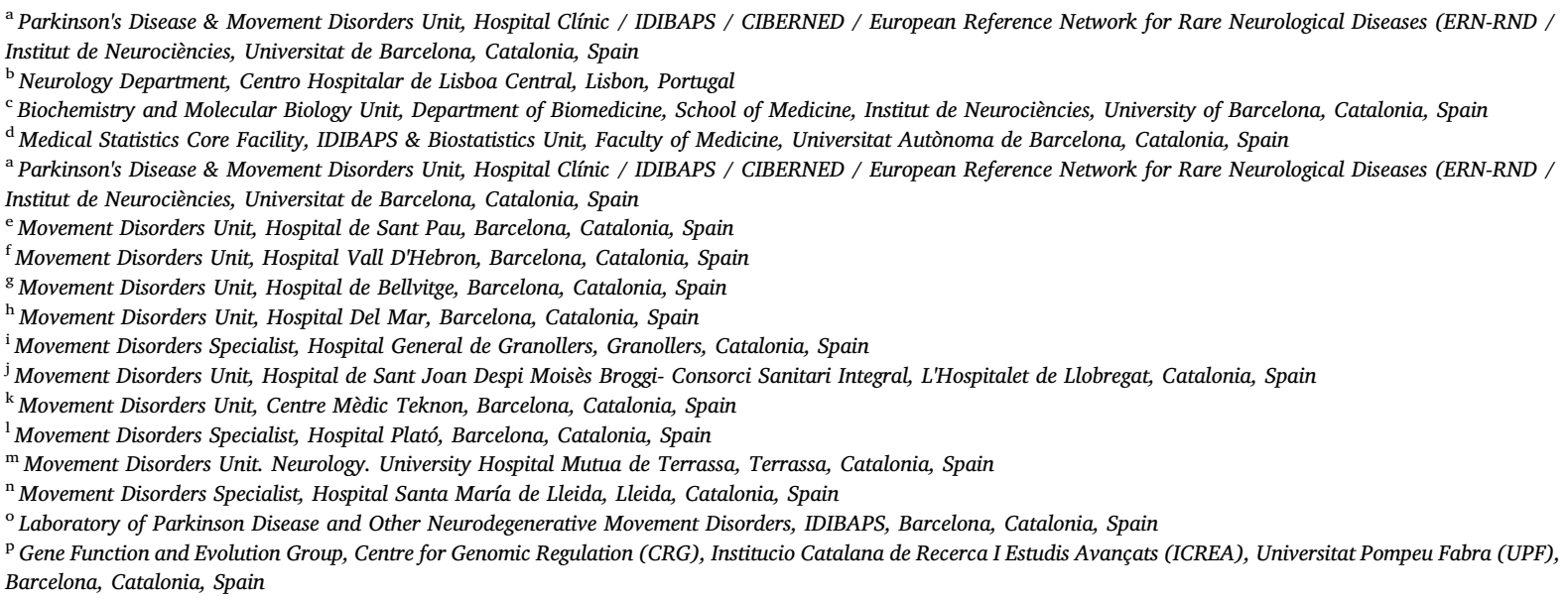

\section{A R T I C L E I N F O}

\section{Keywords:}

Multiple system atrophy

Parkinson's disease

Cerebrospinal fluid

Cytokines

Inflammation

Biomarkers

\begin{abstract}
A B S T R A C T
Introduction: Neuroinflammation is a potential player in neurodegenerative conditions, particularly the aggressive ones, such as multiple system atrophy (MSA). Previous reports on cytokine levels in MSA using serum or cerebrospinal fluid (CSF) have been inconsistent, including small samples and a limited number of cytokines, often without comparison to Parkinson's disease (PD), a main MSA differential diagnosis.

Methods: Cross-sectional study of CSF levels of 38 cytokines using a multiplex assay in 73 participants: 39 MSA patients (19 with parkinsonian type [MSAp], 20 with cerebellar type [MSAc]; 31 probable, 8 possible), 19 PD patients and 15 neurologically unimpaired controls. None of the participants was under non-steroidal antiinflammatory drugs at the time of the lumbar puncture.

Results: There were not significant differences in sex and age among participants. In global non-parametric comparisons FDR-corrected for multiple comparisons, CSF levels of 5 cytokines (FGF-2, IL-10, MCP-3, IL-12p40,
\end{abstract}

\footnotetext{
* Corresponding author. 170 Villarroel street, Neurology Service, Hospital Clinic de Barcelona, 08036, Barcelona, Catalonia, Spain.

${ }^{* *}$ Corresponding author. 170 Villarroel street, Neurology Service, Hospital Clinic de Barcelona, 08036, Barcelona, Catalonia, Spain.

E-mail addresses: ycompta@clinic.cat (Y. Compta), mjmarti@clinic.cat (M.J. Martí).

${ }^{1}$ Both these authors contributed equally to this work.
} 
MDC) differed among the three groups. In pair-wise FDR-corrected non-parametric comparisons 12 cytokines (FGF-2, eotaxin, fractalkine, IFN- $\alpha 2$, IL-10, MCP-3, IL-12p40, MDC, IL-17, IL-7, MIP-1 $\beta$, TNF- $\alpha$ ) were significantly higher in MSA vs. non-MSA cases (PD + controls pooled together). Of these, MCP-3 and MDC were the most significant ones, also differed in MSA vs. PD, and were significant MSA-predictors in binary logistic regression models and ROC curves adjusted for age. CSF levels of fractalkine and MIP-1 $\alpha$ showed a strong and significant positive correlation with UMSARS-2 scores.

Conclusion: Increased CSF levels of cytokines such as MCP-3, MDC, fractalkine and MIP-1 $\alpha$ deserve consideration as potential diagnostic or severity biomarkers of MSA.

\section{Introduction}

Multiple system atrophy (MSA) is a rapidly progressive neurodegenerative condition that can clinically present as parkinsonism (MSAp) or cerebellar syndrome (MSAc), requiring dysautonomia for the clinical diagnosis [1]. Definitive diagnosis necessitates the neuropathological confirmation of glial cell alpha-synuclein inclusions in basal ganglia, brainstem and cerebellar structures [1]. Accordingly, MSA is classified as a synucleinopathy alongside Parkinson's disease (PD) raising the concern that alpha-synuclein biomarkers might fail to differentiate both conditions [2,3]. Thus, lately non-alpha-synuclein biomarkers of MSA such as the light chain of neurofilament and coenzyme Q10 in peripheral blood or cerebrospinal fluid (CSF) [4-8] have been investigated.

Another alternative is that of cytokines as a proxy of inflammatory activity, with the hypothesis that the aggressiveness of MSA might be related to greater neuro-inflammation compared to PD. Still, the interpretation of cytokines changes is challenging, due to their wide range of functions (proinflammatory, anti-inflammatory, unrelated to inflammation, unknown). Moreover, cytokines might show alterations in other aggressive parkinsonisms and be non-specific markers. Up to now, most published studies on cytokines levels in MSA have used peripheral blood $[9,10]$. This biofluid poses the caveat of potential confound by systemic inflammatory processes or drugs, such as non-steroidal antiinflammatory drugs (NSAIDs). Moreover, most of these studies have not compared MSA to PD $[10,11]$. Conversely, CSF theoretically could allow for more specifically assessing intrathecal processes. In the largest CSF report published to date, 27 cytokines were assessed in 20 MSAc cases and compared to 12 subjects with spinocerebellar ataxia (SCA) and 15 non-inflammatory neurological controls, with main findings being a similar proinflammatory profile in MSAc and SCA, associated with the disease course in the case of MSAc.

In view of all this, we hypothesized that CSF cytokines would be differently expressed in MSA subjects, not only relative to neurologically unimpaired controls, but also to PD patients, in spite of both MSA and PD being synucleinopathies.

\section{Methods}

Design and setting: In this convenience cross-sectional pilot study we used the demographic and clinical data along with the CSF samples of patients from the Catalan MSA Registry (CMSAR). Patients were recruited from several neurological centres in Catalonia (population 7.5 million), between March 2015 and February 2018, and diagnosed according to the second consensus statement on MSA diagnosis [12]. The data and CSF samples from PD and control subjects were obtained from the Movement Disorders Biosample Collection of Hospital Clinic de Barcelona (MDBC-HCB), a single centre biorepository consisting of several biosamples including CSF. We have used part of these CSF samples in previous studies $[8,13]$. All the samples from both the CMSAR and the MDBC-HCB were collected, processed and analysed in a centralized way at the coordinating centre (Hospital Clínic de Barcelona). Cases under NSAIDs at the time of lumbar puncture were excluded. Both the CMSAR and the MDBC-HCB along with the present CSF biomarker study have received approval from the competing Ethics Committee. All participants or their representatives signed a written informed consent.

Demographical and clinical data: Movement disorders specialized neurologists (DMG, APS, MJM) collected demographic and clinical variables of all participants through the clinical history, the neurological examination and the motor sections of the Unified Multiple System Atrophy Rating Scale (UMSARS-2) [14] or the Unified PD Rating Scale (UPDRS-3) [15], and the Hoehn and Yahr (H\&Y) staging [16]. The variables considered for this study were age at disease onset and at inclusion, sex, disease duration, levodopa equivalent daily dose (LEDD) [17], and the UMSARS-2 scores.

CSF sample collection: CSF samples were obtained via lumbar puncture at the L2-L3 level with a 22-gauge needle, between 8 and 10 a.m. after overnight fasting and in off-medication condition. The first $2 \mathrm{~mL}$ were used for routine studies. The following $10 \mathrm{~mL}$ were, immediately centrifuged at $4,000 \mathrm{~g}$ for $10 \mathrm{~min}$ and stored at $-80^{\circ} \mathrm{C}$ in $300 \mu \mathrm{L}$ polypropylene aliquots until analysis. Only CSF samples with an erythrocyte count of 0 were included.

CSF cytokines analysis: A premixed multiplex 38-bead assay, the Human Cytokine and Chemokine Panel (HCYTMAG-60K-PX38; Milliplex, Millipore, Billerica, MA, USA), was performed according to the manufacturer's instructions using undiluted samples in a Luminex 200 TM analyzer. All CSF samples were analysed in duplicate. The concentration of each given cytokine for each study participant was calculated as the mean of both duplicates, as usual. We determined 38 cytokines and chemokines: soluble CD40 ligand (sCD40L), epidermal growth factor (EGF), eotaxin, fibroblast growth factor 2 (FGF-2), FMSlike tyrosine kinase 3 ligand (Flt-3L), fractalkine, granulocyte colony stimulating factor (G-CSF), GM-CSF, growth-regulated oncogene (GRO), IFN- $\alpha 2$, IFN- $\gamma$, IL-1 $\alpha$, IL-1 $\beta$, IL-1ra, IL-2, IL-3, IL-4, IL-5, IL-6, IL7, IL-8, IL-9, IL-10, IL-12p40, IL-12p70, IL-13, IL-15, IL-17A, IFN- $\gamma$-induced protein 10 (IP-10/CXCL10), monocyte chemotactic proteins-1 and 3 (MCP-1/CCL2, MCP-3/CCL7), macrophage-derived chemokine (MDC/CCL22), macrophage inflammatory proteins $1 \alpha$ and $1 \beta$ (MIP-1 $\alpha$ / CCL3, MIP-1 $\beta / C C L 4)$, transforming growth factor $\alpha$ (TGF- $\alpha$ ), TNF- $\alpha$ and $\beta$, and vascular endothelial growth factor (VEGF). Data acquisition was performed using the Luminex ${ }^{\circ}$ XPONENT $^{\circ}$ software. For statistical analyses purposes, cases with undetectable levels in a given cytokine were assigned the lowest value of the cohort for that particular cytokine divided by two in order to be included in the comparative analyses. Sample wells with bead counts below 35 were excluded from analysis due to lesser reliability in their signal. Likewise, duplicates with a coefficient of variation (CV) $>25 \%$ were excluded. Additionally, we checked that there were not large differences in the proportion of undetectable cases or cases with $<35$ beads or with $\mathrm{CV}>25 \%$ among the study groups.

Statistical plan and analysis: No formal power calculations were carried out beforehand due to the rarity of MSA, with the number of participants (greater than that from previous positive studies) being deemed reasonable. Categorical variables are expressed as frequencies and were compared using the $\chi 2$ square test or the Fisher exact test. Continuous variables are expressed as median and interquartile range (IQR). Kruskal-Wallis test was used for global comparisons followed by Mann-Whitney's $U$ test for pair-wise comparisons (MSA vs. PD, MSA vs. controls, MSA vs. non-MSA (PD + controls), PD vs. controls and also MSA-p vs. PD). All analyses (both comparisons, either global or pair- 


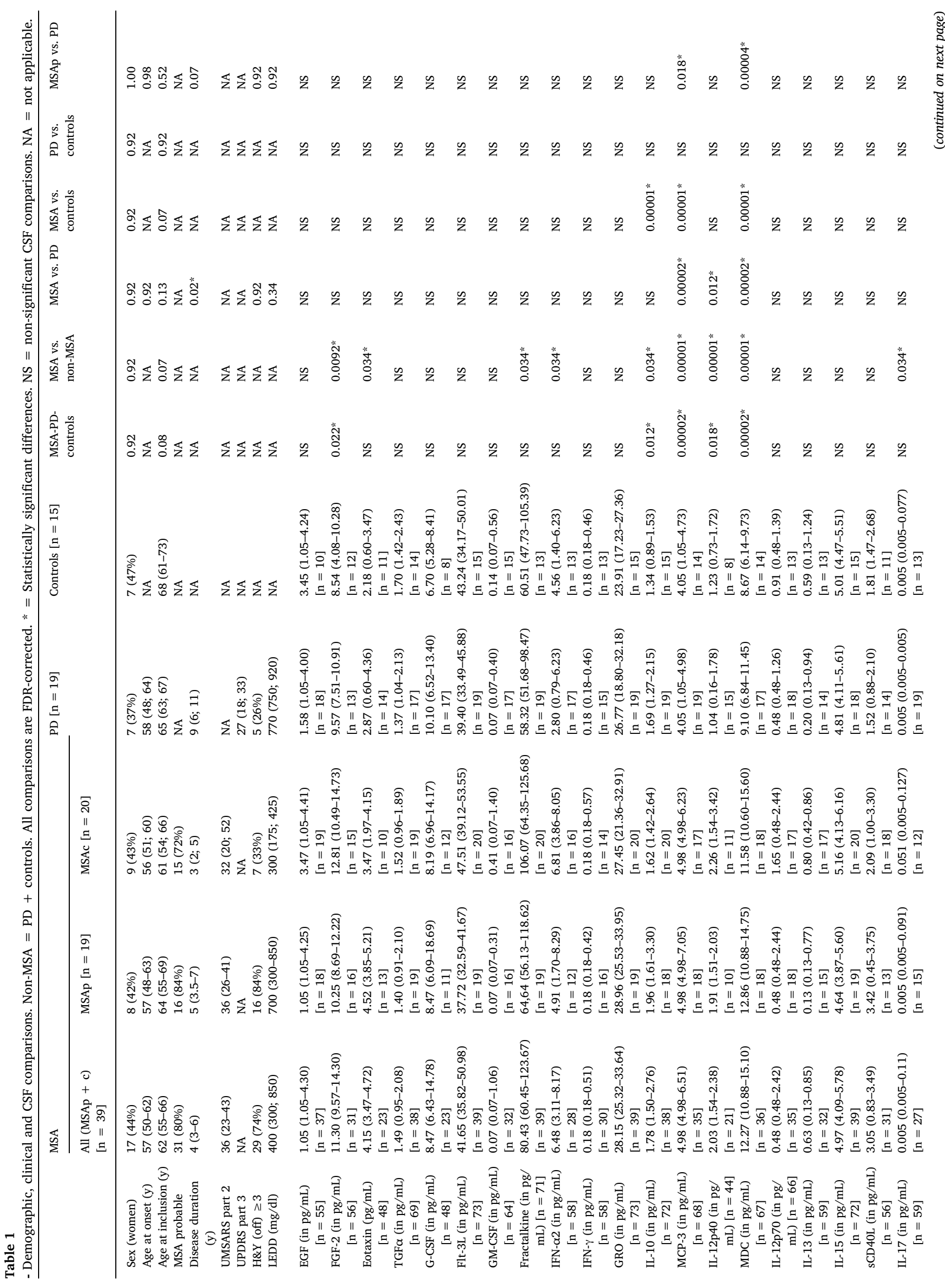




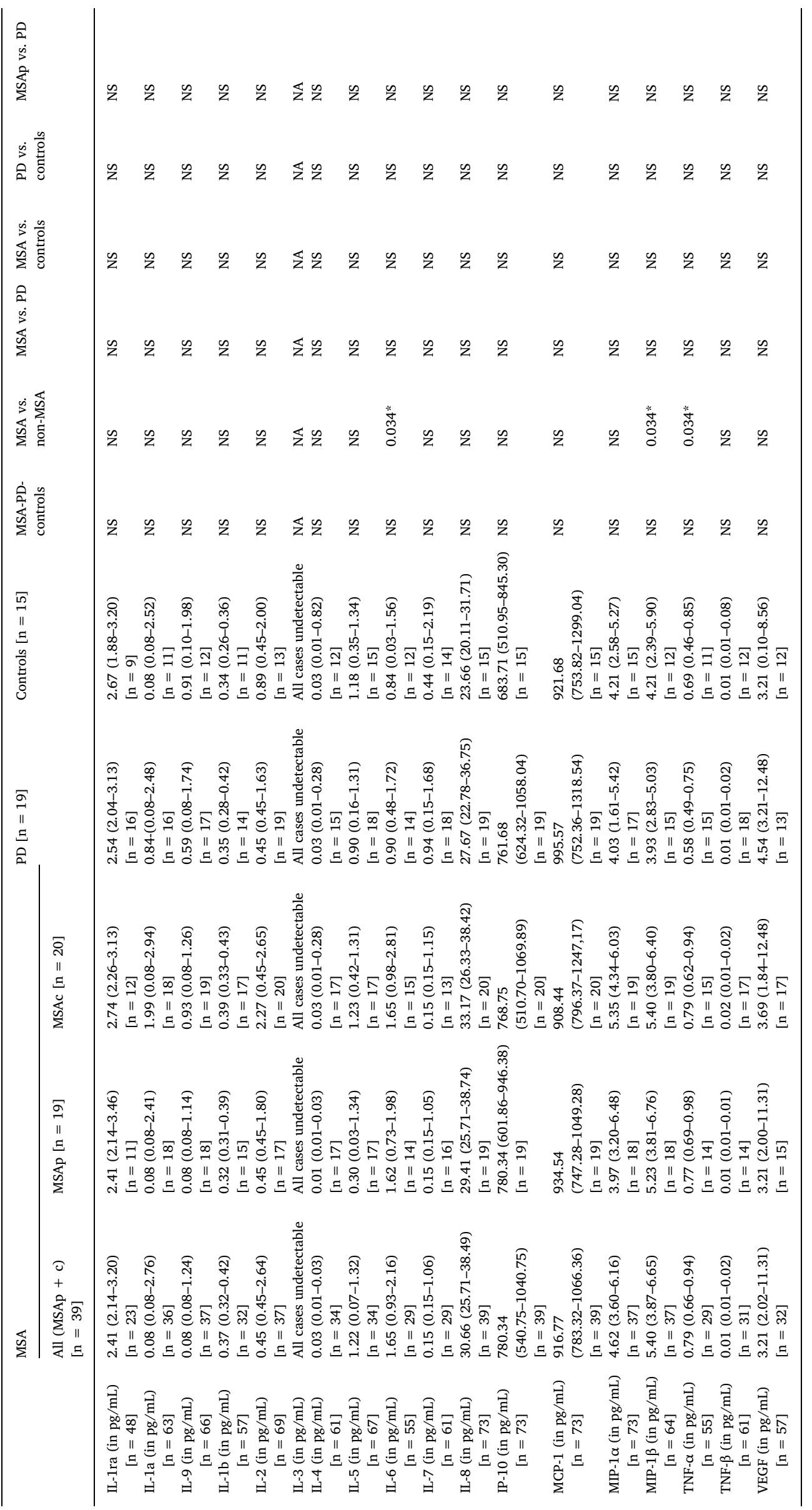


wise, and correlations) were corrected for multiple comparisons by means of false discovery rate (FDR) [18] considering the number of demographic and clinical variables in the descriptive part, and the number of assessable cytokines $(n=37$, since one cytokine was undetectable in all cases, see further on) in the part of CSF cytokines levels. Subsequently, we identified the most significant, corrected CSF variables with data available for the larger part of the cohort as potential MSA predictors (or independent variables) in binary logistic
A
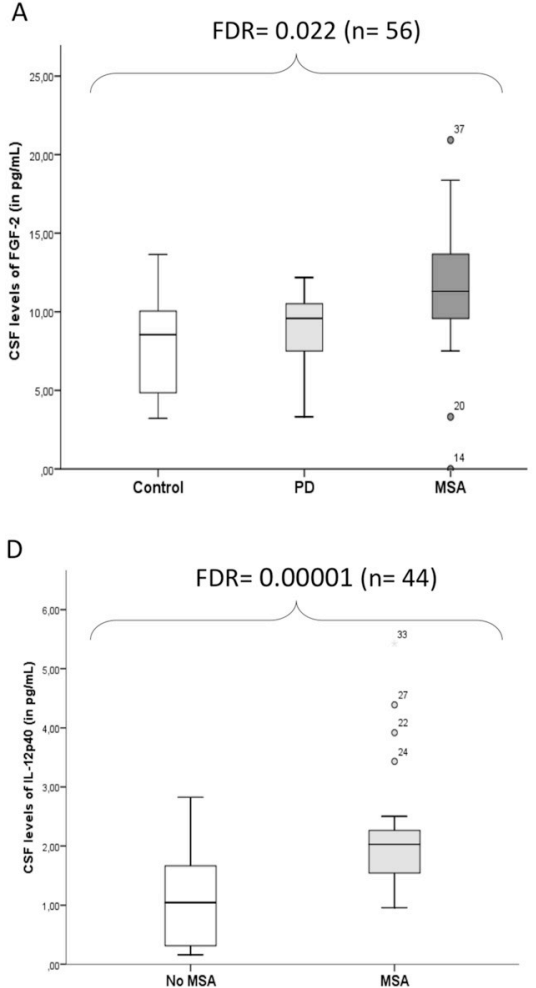

G

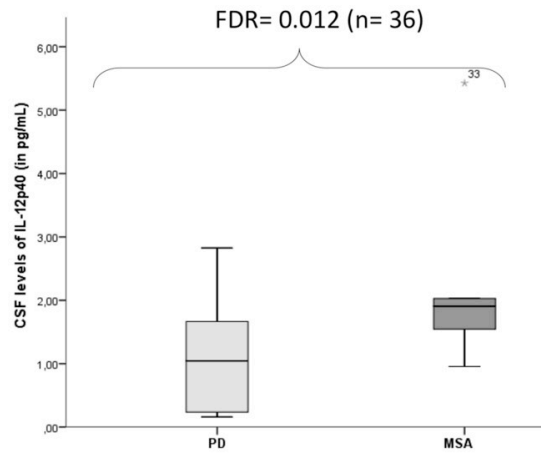

B

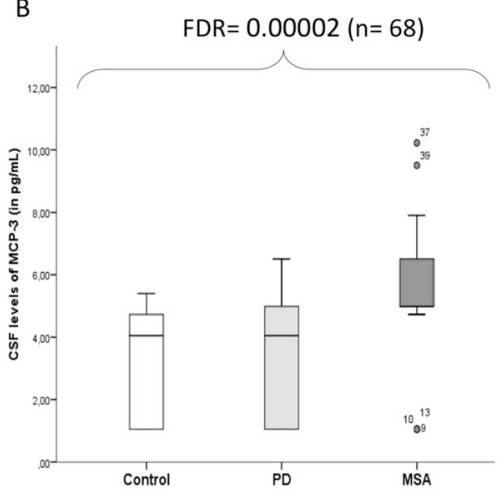

$\mathrm{E}$

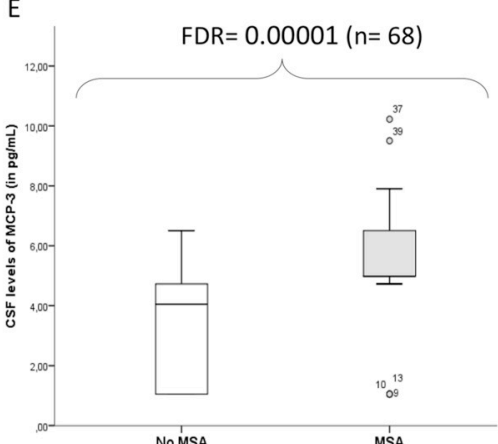

$\mathrm{H}$

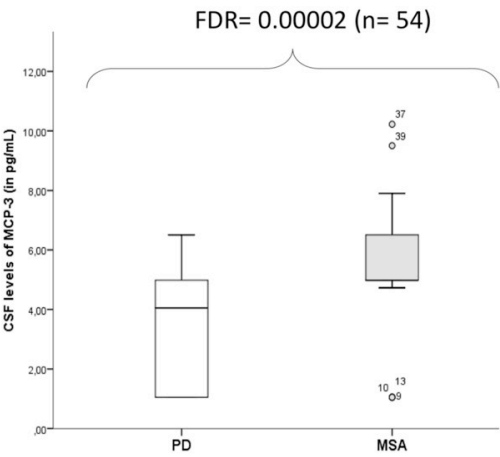

C
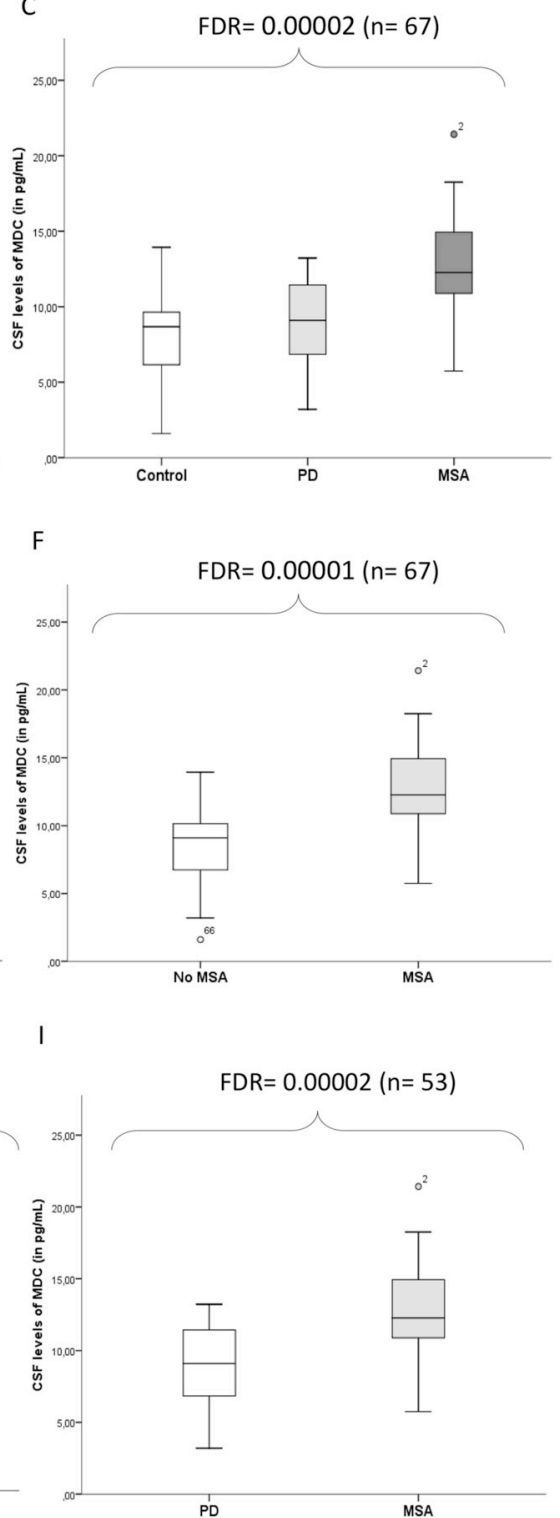

J

K
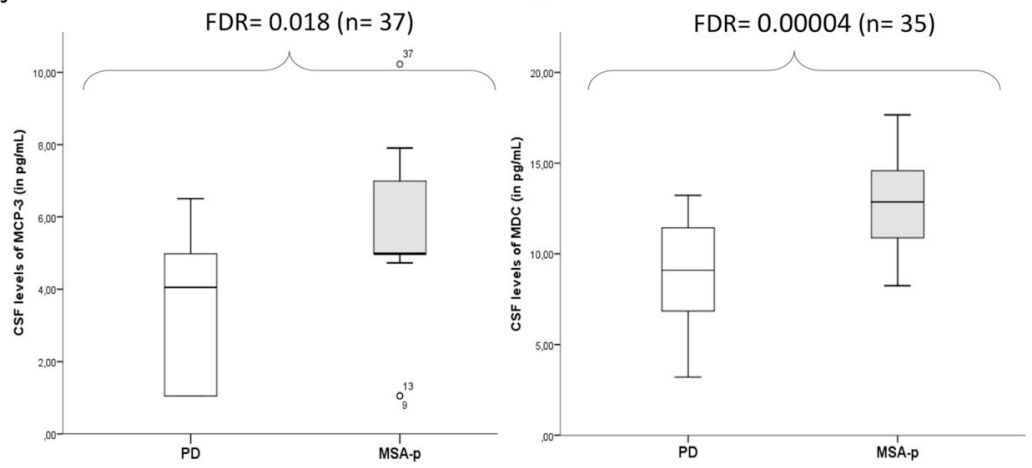

Fig. 1. Box plots of CSF levels (in pg/mL) of FGF-2 [A], MCP-3 [B] and MDC [C] in global comparisons (MSA, PD, controls), of IL-12p40 [D], MCP-3 [E] and MDC [F] in pair-wise comparison of MSA vs. non-MSA (PD + controls), as well as when comparing MSA vs. PD [G, H, I], and finally of MCP-3 and MDC in the pair-wise comparison of MSA-p vs. PD [J, K]. 
regression models with MSA diagnosis as outcome (dependent variable). In these models the alternative outcome to MSA was either nonMSA (PD patients + controls, as the cases without the target diagnostic, as done in previous biomarkers studies) $[2,8]$ or PD (the obvious differential diagnosis for MSA and particularly MSA-p). Potential modifiers, such as age and sex were included in the models, limiting the number of covariates according to the size of the tested sample to avoid overfitting. These binary logistic regression models resulted in the respective odds ratios (OR) and their 95\% confidence intervals (95\%CIs). We used the estimated probabilities from the binary logistic regression model combining the CSF cytokines most significantly associated with MSA and with data available for the better part of the cohort in a ROC curve analysis to discriminate MSA vs. non-MSA (PD + controls) with the respective area under the curve (AUCs) and its 95\%CI. Additionally, we run ROC curves for individual CSF levels of significant cytokines for the discrimination of MSA from PD, and of MSA-p from PD. Linear correlations between CSF cytokines levels and quantitative demographic and clinical data were further explored with Spearman's correlation (also FDR-corrected for multiple comparisons). All statistical tests were two-tailed, with significance threshold set at a p-value $\leq 0.05$. Analyses were performed using IBM SPSS Statistics software, version 24.0 (Armonk, NY: IBM Corp).

\section{Results}

Demographic and clinical comparisons (Table 1): We included 39 MSA patients (19 with parkinsonian type [MSAp], 20 with cerebellar type [MSAc]; 31 probable, 8 possible, with all of them having been reclassified as probable thereafter at follow-up), 19 PD patients and 15 neurologically unimpaired controls. There were no significant differences in sex and age. Disease duration was greater in PD vs. MSA. Side effects of lumbar puncture were mild (transient headache, puncture site pain) and rare (5\%).

Multiplex assay performance: IL-3 was below the detection range in all samples. Flt-3L, GRO, IL8, IP10 and MCP-1 were assessable in all participants. There were no significant differences in the proportion of undetectable cases and of those with $<35$ beads or CV $>25 \%$ among groups except for eotaxin, MCP-3 and VEGF (more frequently undetectable in PD and controls than in MSA), IL-2 (more cases with $<35$ beads in MSA) and IL-1ra (more cases with CV > 25\% in MSA) (Data not shown).

Comparisons of CSF levels of cytokines (Table 1; Fig. 1; Suppl. Table 1): In global non-parametric comparisons (Kruskal-Wallis test) with FDR-correction for multiple comparisons, CSF levels of 5 cytokines (FGF-2, IL-10, MCP-3, IL-12p40, MDC) differed among MSA, PD and controls. In pair-wise comparison (Mann-Whitney's $U$ test) with FDRcorrection for multiple comparisons, there were 12 cytokines (FGF-2, eotaxin, fractalkine, IFN- $\alpha 2$, IL-10, MCP-3, IL-12p40, MDC, IL-17, IL-7, MIP-1 $\beta$, TNF- $\alpha$ ) with significantly higher CSF levels in MSA than in non-MSA (PD + control) cases. Of these, MCP-3, IL-12p40 and MDC had FDR-corrected significantly higher CSF levels in MSA compared to PD (with MCP-3 and MDC also retaining the significant difference in the comparison limited to MSA-p vs. PD). When comparing MSA to controls, IL-10, MCP-3 and MDC had FDR-corrected significantly higher CSF levels in the former relative to the latter group. There were no significant FDR-corrected differences in the CSF levels of any cytokine in PD patients vs. controls. There were no FDR-corrected significant CSF cytokines differences between MSAp and MSAc either (Data not shown).

Adjusted binary logistic regression models and ROC curves (Table 2; Fig. 2A; Suppl. Fig. 1A-B): CSF MCP-3 and MDC levels (Fig. 1) were significantly associated with MSA vs. non-MSA in the respective binary logistic regression models adjusting for age and sex. In a model combining age at inclusion and both these cytokines, younger age and increasing CSF levels of MCP-3 and MDC were significant predictors of MSA vs. non-MSA. Accordingly, the ROC curve generated by using the estimated probabilities from the aforementioned regression model showed a significant discriminant ability of MSA vs. non-MSA of the combination of decreasing age and increasing CSF levels of MCP-3 and MDC. Additionally, CSF levels of either MCP-3 or MDC were predictive of MSA vs. PD and of MSA-p vs. PD in the respective binary logistic regression models and ROC curves.

Linear correlations in MSA: There were no FDR-corrected significant correlations between CSF levels of cytokines and either age at onset, age

Table 2

Significant binary logistic regression models with different combinations of demographic and CSF variables as predictors (independent variables) and MSA or MSA-p as outcome (dependent variable).

\begin{tabular}{|c|c|c|c|c|}
\hline MSA vs. non-MSA & Odds ratio & \multicolumn{2}{|l|}{$95 \%$ confidence interval } & $\mathrm{p}$-value \\
\hline \multicolumn{5}{|c|}{ Model 1 (MSA, $n=35$; non-MSA, $n=33$ ) [total, $n=68$ ] } \\
\hline Sex (woman) & 0.74 & 0.21 & 2.52 & 0.738 \\
\hline CSF MCP-3 levels (in pg/mL) & 2.19 & 1.44 & 3.33 & $<0.001^{\mathrm{a}}$ \\
\hline \multicolumn{5}{|c|}{ MODEL 2 (MSA, $\mathrm{n}=36$; non-MSA, $\mathrm{n}=31$ ) [total, $\mathrm{n}=67$ ] } \\
\hline Age at inclusion (in years) & 0.85 & 0.76 & 0.94 & $0.003^{\mathrm{a}}$ \\
\hline \multicolumn{5}{|c|}{ MODEL 3 (MSA, $\mathrm{n}=32$; non-MSA, $\mathrm{n}=30$ ) [total, $\mathrm{n}=62$ ] } \\
\hline Age at inclusion (in years) & 0.82 & 0.71 & 0.95 & $0.009^{\mathrm{a}}$ \\
\hline CSF MCP-3 levels (in pg/mL) & 2.05 & 1.28 & 3.29 & $0.003^{\mathrm{a}}$ \\
\hline CSF MDC levels (in $\mathrm{pg} / \mathrm{mL}$ ) & 2.21 & 1.33 & 3.68 & $0.002^{\mathrm{a}}$ \\
\hline \multirow[t]{2}{*}{ MSA vs. PD } & Odds ratio & $95 \%$ confidence interval & & $p$-value \\
\hline & & Lower margin & Upper margin & \\
\hline \multicolumn{5}{|c|}{ MODEL 1 (MSA, $\mathrm{n}=35 ; \mathrm{PD}, \mathrm{n}=19$ ) [total, $\mathrm{n}=54$ ] } \\
\hline \multicolumn{5}{|c|}{ MODEL 1 (MSA-p, $\mathrm{n}=18 ; \mathrm{PD}, \mathrm{n}=19$ ) [total, $\mathrm{n}=37$ ] } \\
\hline CSF MCP-3 levels (in pg/mL) & 1.75 & 1.15 & 2.67 & $0.009^{\mathrm{a}}$ \\
\hline \multicolumn{5}{|c|}{ MODEL 2 (MSA-p, $\mathrm{n}=18 ; \mathrm{PD}, \mathrm{n}=17$ ) [total, $\mathrm{n}=35$ ] } \\
\hline CSF MDC levels (in pg/mL) & 1.80 & 1.19 & 2.74 & $0.006^{\mathrm{a}}$ \\
\hline
\end{tabular}

a $=$ significant association. Note that for quantitative variables the ORs indicate the change in risk per each measure unit increase or decrease in the predictor. 
A

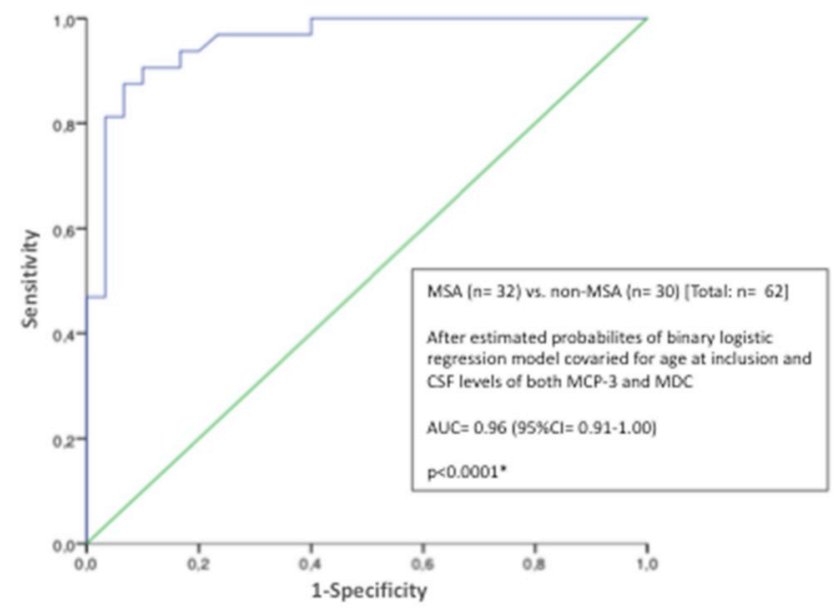

B

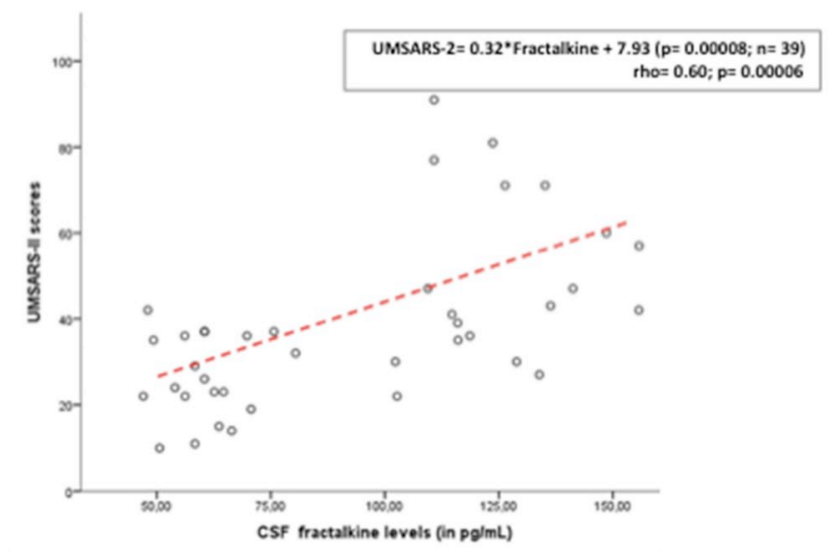

C

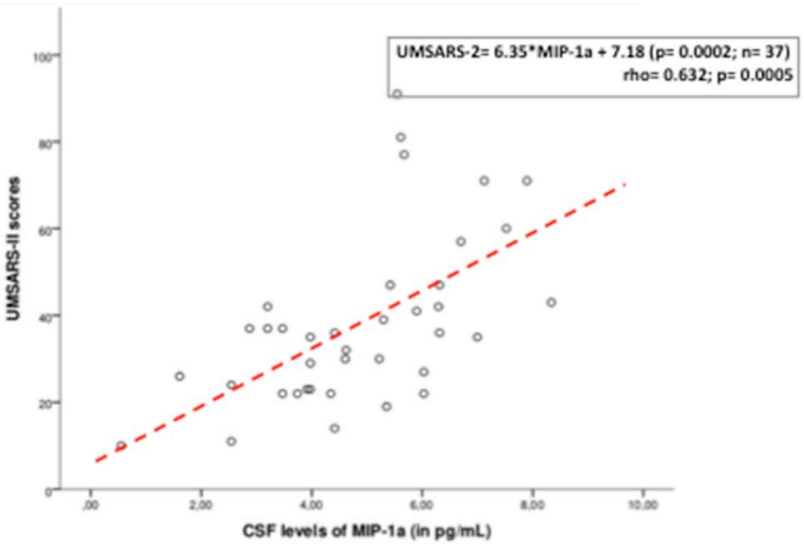

Fig. 2. [A] ROC curve generated with the estimated probabilities from the binary logistic regression model including age at inclusion and CSF levels of both MCP-3 and MDC in combination to discriminate MSA from non-MSA; [B] FDR-corrected significant positive linear correlation between the CSF levels of fractalkine and UMSARS-2 scores; [C] FDR-corrected significant positive linear correlation between the CSF levels of MIP-1 $\alpha$ and UMSARS- 2 scores.

at inclusion, disease duration, or LEDD.

Conversely, the CSF levels of up to 17 of the 38 tested cytokines (including 4 of the cytokines significantly differing between MSA and non-MSA: FGF-2, fractalkine, IFN- $\alpha 2$ and IL-10) showed FDR-corrected significant positive linear correlations with the UMSARS-2 scores (Suppl. Table 2). These correlations were particularly significant in the case of the CSF levels of fractalkine and also MIP-1 $\alpha$ (Fig. 2B-C).

Additionally, there were significant FDR-corrected correlations among several CSF cytokines levels in MSA patients. Those were mostly positive correlations, as in the case of FGF-2 with both MCP-3 and IL-
$12 \mathrm{p} 40$, of fractalkine with both IFN- $\alpha 2$ and IL-7, of IFN- $\alpha 2$ with both IL7 and IL-17, and of MIP-1 $\beta$ with both MCP-3 and TNF- $\alpha$ (Suppl. Table 3).

\section{Discussion}

In this study CSF levels of several cytokines were increased in MSA vs. PD and controls, although with variability in the detectability and reliability of these measurements. Still, 12 of the 38 tested cytokines were significantly increased in MSA compared to non-MSA cases, with two of these (MCP-3 and MDC) being also significantly increased in the pair-wise comparisons of MSA and MSA-p vs. PD and when comparing MSA with controls, being significant predictors of MSA in binary regression models and ROC curves too. CSF levels of fractalkine and of MIP-1 $\alpha$ also showed significant and positive correlations with the UMSARS-2 scores. All this suggests that these CSF cytokines levels could be diagnostic or severity markers for MSA and assist the differentiation between MSA and PD, which is challenging with current alpha-synuclein biomarkers.

The interpretation of our findings, consisting of a mixture of proand anti-inflammatory cytokines, is largely speculative from a mechanistic point of view. Still, and not forgetting that these are "markers" and not "makers", it cannot be ruled out that at the time of CSF collection, there was a mixed expression of pro-inflammatory cytokines (pointed to play a role early in the disease) and anti-inflammatory ones (which might be the result of secondary compensatory mechanisms) [19]. In fact, our top significant MSA predictors (CSF levels of MCP-3 and MDC) have a pro-inflammatory and an anti-inflammatory profile each.

We are unaware of previous studies having assessed MCP-3 (or CCL7) in MSA. Nevertheless, this cytokine that has pro-inflammatory and chemotactic properties and that was markedly increased in CSF of our MSA patients compared to both PD patients and controls, has been previously pointed to be a differentiation factor for midbrain dopaminergic precursors and neurons and might therefore be connected with degenerative parkinsonisms such as MSA [20].

Our other top candidate CSF cytokine to differentiate MSA from non-MSA, MDC, also known as C-C motif chemokine ligand 22 (CCL22), and its receptor have been associated with recruitment of Tcells in chronic inflammatory processes. MDC has been implicated in a number of diseases, including experimental autoimmune encephalitis [21]. Ours is the first study measuring CSF levels of MDC in MSA.

Another of the other CSF cytokines significantly increased in our MSA patients, IL-10 (an anti-inflammatory cytokine involved in the interactions between microglia, astrocytes and neurons) [22], only differed between MSA patients and controls, but not between MSA and PD participants, who had non-significantly higher levels than controls. This finding is similar to those from two previous studies using serum instead of CSF $[8,23]$. Specifically in the study by Brodacki et al., IL-10 levels in atypical parkinsonisms were in between those of PD and controls, but only two patients with MSA were included [8]. Another study found CSF levels of IL-10 to be lower in MSA-C and spinocerebellar ataxias (SCA), but compared to other non-inflammatory neurological disorders, rather than non-neurological controls or other parkinsonisms (neither MSA-P nor PD were included) [11]. As elevated IL-10 is anti-inflammatory and can be potentially neuroprotective in the acute phase of ischaemic stroke [24], its increase in MSA and PD may reflect compensatory mechanisms.

Of the other CSF cytokines significantly increased in MSA, FGF-2 and IL-12p40 showed remarkable differences in global and MSA vs. non-MSA comparisons, respectively. The association of FGF-2 and IL12p40 with synucleinopathies such as MSA is biologically plausible. Regarding FGF, it has been found to promote alpha-synuclein expression [25], but also to protect the nigrostriatal system from 6-hydroxidopamine [26]; hence, its increase in MSA might be either a result of the synucleinopathy itself or a compensatory attempt to protect 
neurons. IL-12p40 on the other hand has been recently associated with PD subtypes, albeit with higher levels implying a more benign phenotype [27], whereas in our case its levels were increased in MSA, which is more aggressive than $\mathrm{PD}$.

Fractalkine (C-X3-C-motif chemokine ligand 1; CX3CL1), which showed a strong positive correlation with high (i.e., worse) UMSARS-2 scores, is a chemokine constitutively expressed in neurons that, together with its receptor CX3CR1 (expressed in microglia), is thought to play a crucial role in mediating neuron-microglia communications in the CNS. Fractalkine has been reported to have both neuroprotective/ anti-inflammatory and neurotoxic/proinflammatory properties in several experimental models of neurological disorders. Such dual activities might be dependent on disease stage and the degree of microglial activation [28-31]. In contrast to our results, CSF levels of fractalkine did not differ between patients with PD, MSA and controls in a previous study by Shi and colleagues, where CSF levels of Flt-L3 were significantly reduced in MSA vs. PD [32] unlike in our study. Potential explanations for these discrepancies are different methodological techniques and populations, as well as the design more focused in PD in Shi et al.'s study and in MSA in our case. Two other studies have not found differences in Flt-L3 either [33,34].

While MIP-1 $\beta$ was among the CSF cytokines increased in MSA (but not the most significant one), the other cytokine strongly correlating with UMSARS-2 scores along with fractalkine was MIP-1 $\alpha$ (macrophage inflammatory protein $1 \alpha$, or CCL3). This pro-inflammatory cytokine might be of potential interest since its levels were increased in a recently reported mouse model of MSA [35]. However, to the best of our knowledge its levels in a biofluid from MSA patients had not been previously reported to correlate with clinical measures of these patients as in our study.

The main comparison of our study is to be made with the one by Yamasaki et al. [11]. As mentioned before, in that study 27 cytokines were assessed only in MSAc and only compared to SCA and neurological controls, and whether or not samples were tested in duplicate was not specified. The main results were increases in CSF levels of IL-6, IL-7, IL-12, IL-13, and GM, and decreases in FGF-2, VEGF, IL-1 $\beta$, IL-2, IL-4, IL-5, IL-8, IL-10, IL-15, MIP-1 $\beta$, and TNF- $\alpha$ in MSA-C and SCA vs. neurological controls [11]. Additionally, MCP-1 negatively correlated with disease duration and IL8 and IL-1 with the UMSARS-2. CSF levels of IL6 were associated with the hot cross bun sign in brain MRI [11]. In contrast to Yamasaki et al., and as mentioned above we have found positive correlations between UMSARS-2 and CSF levels of both antiinflammatory (fractalkine) and pro-inflammatory (MIP-1 $\alpha$ ) cytokines, suggesting a link between inflammation and clinical severity of the degenerative parkinsonism. These differences in results might be due to different ethnicities and patients (only MSAc vs. SCA in Yamasaki et al.'s; both MSAp and MSAc vs. PD in our study) indicating that more work needs to be done in this area.

This study is not without limitations. MSA participants had established disease limiting our interpretation to advanced rather than early cases. The PD and control groups were relatively small and, in the case of PD, with longer disease duration than MSA. Thus, we cannot exclude bias due to disease duration, which could not be included as a covariate in regression models due to insufficient sample size. Nevertheless, disease duration did not correlate with the CSF levels of any of the tested cytokines. This study lacks a validation cohort, and has no neuropathological confirmation of most cases. However, all three cases autopsied since study completion have been confirmed as MSA. The regional setting compromises generalization as variation among ethnicities might be relevant (for example compared to prior East Asian studies). Furthermore, CSF was collected only at baseline, and all analyses are cross-sectional. Finally, we included eight possible MSA cases, but all have been thereafter reclassified as probable. Hence, if any, the effect of inclusion of possible MSA cases might have been an under- rather than an overestimation of CSF cytokines differences.

Our study also has strengths. It is the largest MSA sample with the greatest number of CSF cytokines assessed to date. We investigated both MSAp and MSAc and compared them not only to PD (a main differential diagnosis considering that both conditions are synucleinopathies), but also to a group of individuals without known neuropsychiatric pathology. Another advantage over part of previous studies is the determination of cytokines in CSF and the exclusion of cases treated with NSAIDs. Moreover, the collection and handling of the samples was centralized. Laboratory technicians who run the multiplex technique were blind to the clinical diagnoses. Additionally, the findings were corrected for multiple comparisons and adjusted for potential modifiers.

In summary, our findings suggest that several CSF levels of cytokines are increased in MSA, with the combination of age at assessment and CSF levels of MCP-3 and MDC showing potential as diagnostic markers of MSA, while CSF levels of fractalkine and MIP-1 $\alpha$ strongly correlated with UMSARS-2 scores. Larger studies with validation cohorts and longitudinal assessments, as well as comparison to other atypical parkinsonisms, are warranted to further evaluate the CSF levels of these cytokines as diagnostic or severity markers in MSA.

\section{Authors' roles}

All authors contributed to one or more of the following: (1) the conception and design of the study, or acquisition of data, or analysis and interpretation of data, (2) drafting the article or revising it critically for important intellectual content, (3) final approval of the version to be submitted.

\section{Financial disclosures}

Yaroslau Compta has received funding, research support and/or honoraria from the IISCIII (PI17/00096), European Commission H2020 (1PI043760), UCB, Teva, Medtronic, Abbvie, Merz, Bial, Zambon and Alter.

Sara P. Dias received a research grant from the European Society of Clinical Microbiology and Infectious Diseases and support from Zambon for participation in a conference.

Darly M. Giraldo reports no disclosures and no conflict of interest.

Alexandra Pérez-Soriano reports no disclosures and no conflict of interest.

Esteban Muñoz reports no disclosures and no conflict of interest.

Josep Saura has received funding from Instituto de Salud Carlos III (PI14/302 and AC14/00016).

Manel Fernández reports no disclosures and no conflict of interest. Paloma Bravo reports no disclosures and no conflict of interest.

Ana Cámara has received funding from European Commission H2020 (PI043760), Abbvie and Medtronic.

Marta Pulido-Salgado is funded through the ISCIII FIS grant (PI17/ 00096).

Cèlia Painous reports no disclosures and no conflict of interest.

José Ríos reports no disclosures and no conflict of interest.

María José Martí received honoraria for advice and lecture from Abbvie, Bial and Merzt Pharma and grants from Michael J. Fox Foundation for Parkinson Disease (MJFF, USA): MJF_PPMI_10_001, PI044024; Fondo de Investigaciones Sanitarias of Spain (FIS PI17/ 00096) and from Generalitat de Catalunya (AGAUR Exp 2017 SGR 1502).

Javier Pagonabarraga reports no disclosures and no conflict of interest.

Francesc Valldeoriola has received funding and honoraria and/or has acted as advisor for Medtronic, Boston, Teva, Zambon and Abbvie.

Jorge Hernández-Vara has received travel bursaries and educational grants from Abbvie. Has received honoraria for educational presentations from Abbvie, Teva, Bial, Zambon, Italfarmaco and SanofiGenzyme.

Serge Jauma Classen reports no disclosures and no conflict of 
interest.

Victor Puente has served as consultant for Abbvie and Zambon; has received grant/research support from Abbvie.

Claustre Pont-Sunyer reports no disclosures and no conflict of interest.

Núria Caballol has received honoraria from Bial, Italfármaco, Qualigen, Zambon, UCB, Teva and KRKA and sponsorship from Zambon, TEVA and Abbvie for attending medical conferences.

Eduardo Tolosa received honoraria for consultancy from Novartis, TEVA, Bial, Accorda, Boehringer Ingelheim, UCB, Solvay, Lundbeck and BIOGEN and has received funding for research from Spanish Network for Research on Neurodegenerative Disorders (CIBERNED)- Instituto Carlos III (ISCIII), and The Michael J. Fox Foundation for Parkinson's Research (MJFF, USA).

Angels Bayes reports no disclosures and no conflict of interest.

Jaume Campdelacreu reports no disclosures and no conflict of interest.

Oriol de Fàbregues has received honoraria for educational presentations and advice service by Abbvie, UCB Pharma, Zambon, Bial and Teva.

Asunción Ávila has received honoraria from Zambon, Qualigen, and Teva, and sponsorship from Zambon and Abbvie for attending conferences.

Matilde Calopa has received honoraria from Bial, UCB and Abbvie. Carles Gaig reports no disclosures and no conflict of interest. Neus Fabregat reports no disclosures and no conflict of interest. Pau Pastor reports no disclosures and no conflict of interest. Miquel Aguilar reports no disclosures and no conflict of interest. Montserrat Pujol reports no disclosures and no conflict of interest. Almudena Sánchez reports no disclosures and no conflict of interest. Lluís Planellas reports no disclosures and no conflict of interest. Mario Ezquerra reports no disclosures and no conflict of interest. Rubén Fernández-Santiago reports no disclosures and no conflict of interest.

Teresa Botta reports no disclosures and no conflict of interest.

Gian Tartaglia reports no disclosures and no conflict of interest.

\section{Financial disclosure and funding source}

None of the authors has any conflict of interest relevant to the topic of this manuscript; the "Marató de TV3 Foundation" has funded this study (PI043296; PI: Dr. M.J. Marti).

\section{Acknowledgements \& Funding}

We are most grateful to all the participants, as without their generosity none of this research would have been possible. We also acknowledge technical assistance by Mr. Óscar Rogero \& Daniel Terribas (Merck Chemicals and Life Science, Darmstad, Germany). This study has been funded by Fundació la Marató de TV3 (PI043296; principal investigator: Dr. M.J. Marti). Our institution is supported by the CERCA programme of the Generalitat de Catalunya.

\section{Appendix A. Supplementary data}

Supplementary data to this article can be found online at https:// doi.org/10.1016/j.parkreldis.2019.05.040.

\section{References}

[1] A. Fanciulli, G.K. Wenning, Multiple-system atrophy, N. Engl. J. Med. 372 (2015) 249-263.

[2] B. Mollenhauer, J.J. Locascio, W. Schulz-Schaeffer, F. Sixel-Döring, C. Trenkwalder, M.G. Schlossmacher, $\alpha$-Synuclein and tau concentrations in cerebrospinal fluid of patients presenting with parkinsonism: a cohort study, Lancet Neurol. 10 (2011) 230-240.

[3] M. Shahnawaz, T. Tokuda, M. Waragai M, N. Mendez, R. Ishii, C. Trenkwalder, et al., Development of a biochemical diagnosis of Parkinson disease by detection of $\alpha$-synuclein misfolded aggregates in cerebrospinal fluid, JAMA Neurol. 74 (2017) $163-172$.

[4] O. Hansson, S. Janelidze, S. Hall, N. Magdalinou, A.J. Lees, U. Andreasson, et al., Blood-based NfL: a biomarker for differential diagnosis of parkinsonian disorder, Neurology 74 (2017) 930-937.

[5] X. Hu, Y. Yang, D. Gong, Cerebrospinal fluid levels of neurofilament light chain in multiple system atrophy relative to Parkinson's disease: a meta-analysis, Neurol. Sci. 38 (2017) 407-414.

[6] T. Kasai, T. Tokuda, T. Ohmichi, R. Ishii, H. Tatebe, M. Nakagawa, T. Mizuno, Serum levels of coenzyme Q10 in patients with multiple system Atrophy, PLoS One 11 (2016) e0147574.

[7] J. Mitsui, T. Matsukawa, T. Yasuda, H. Ishiura, S. Tsuji, Plasma coenzyme Q10 levels in patients with multiple system atrophy, JAMA Neurol. 73 (2016) 977-980.

[8] Y. Compta, D.M. Giraldo, E. Muñoz, F. Antonelli, M. Fernández, P. Bravo, et al., CatalanMSA Registry (CMSAR), Cerebrospinal fluid levels of coenzyme Q10 are reduced in multiple system atrophy, Park. Relat. Disord. 46 (2018) 16-23.

[9] B. Brodacki, J. Staszewski, B. Toczyłowska, E. Kozłowska, N. Drela, M. Chalimoniuk, A. Stepien, Serum interleukin (IL-2, IL-10, IL-6, IL-4), TNFalpha, and INFgamma concentrations are elevated in patients with atypical and idiopathic parkinsonism, Neurosci. Lett. 441 (2008) 158-162.

[10] E. Kaufman, S. Hall, Y. Surova, H. Widner, O. Hansson, D. Lindqvist, Proinflammatory cytokines are elevated in serum of patients with multiple system atrophy, PLoS One 8 (2013) e62354.

[11] R. Yamasaki, H. Yamaguchi, T. Matsushita, T. Fujii, A. Hiwatashi, J.I. Kira, Early strong intrathecal inflammation in cerebellar type multiple system atrophy by cerebrospinal fluid cytokine/chemokine profiles: a case control study, J. Neuroinflammation 14 (2017) 89.

[12] S. Gilman, G.K. Wenning, P.A. Low, D.J. Brooks, C.J. Mathias, J.Q. Trojanowski, et al., Second consensus statement on the diagnosis of multiple system atrophy, Neurology 71 (2008) 670-676.

[13] Y. Compta, M.J. Martí, N. Ibarretxe-Bilbao, C. Junqué, F. Valldeoriola, E. Muñoz, et al., Cerebrospinal tau, phospho-tau, and beta-amyloid and neuropsychological functions in Parkinson's disease, Mov. Disord. 24 (2009) 2203-2210.

[14] G.K. Wenning, F. Tison, K. Seppi, C. Sampaio, A. Diem, F. Yekhlef, et al., Multiple system Atrophy study group. Development and validation of the unified multiple system Atrophy rating Scale (UMSARS), Mov. Disord. 19 (2004) 1391-1402.

[15] S. Fahn, R.L. Elton, Members of the UPDRS development committee, unified Parkinson's disease rating Scale, in: S. Fahn, C.D. Marsden, D.B. Calne, A. Lieberman (Eds.), Recent Developments in Parkinson's Disease, McMillan Health Care Information, Florham Park, NJ, 1987, pp. 153-163.

[16] M.M. Hoehn, M.D. Yahr, M.D. Parkinsonism, onset, progression, and mortality Parkinsonism: onset, progression, and mortality, Neurology 17 (1967) 427-442.

[17] R. Wenzelburger, B.R. Zhang, S. Pohle, S. Klebe, D. Lorenz, J. Herzog, et al., Force overflow and levodopa-induced dyskinesias in Parkinson's disease, Brain 125 (2002) 871-879.

[18] Y. Benjamini, Y. Hochberg, Controlling the false discovery rate: a practical and powerful approach to multiple testing, J. R. Stat. Soc. Ser. B 57 (1995) 289-300.

[19] N. Stefanova, M. Reindl, M. Neumann, P.J. Kahle, W. Poewe, G.K. Wenning, Microglial activation mediates neurodegeneration related to oligodendroglial alpha-synucleinopathy: implications for multiple system atrophy, Mov. Disord. 22 (2007) 2196-2203.

[20] L.C. Edman, H. Mira, E. Arenas, The beta-chemokines CCL2 and CCL7 are two novel differentiation factors for midbrain dopaminergic precursors and neurons, Exp. Cell Res. 314 (2008) 2123-2130.

[21] R.-N.E. Dogan, N. Long, E. Forde, K. Dennis, A.P. Kohm, S.D. Miller, W.J. Karpus, CCL22 regulates experimental autoimmune encephalomyelitis by controlling inflammatory macrophage accumulation and effector function, J. Leukoc. Biol. 89 (2011) 93-104.

[22] D. Lobo-Silva, G.M. Carriche, A.G. Castro, S. Roque, M. Saraiva, Balancing the immune response in the brain: IL-10 and its regulation, J. Neuroinflammation 13 (2016) 297.

[23] M. Rentzos, C. Nikolaou, E. Andreadou, G.P. Paraskevas, A. Rombos, M. Zoga, et al., Circulating interleukin-10 and interleukin-12 in Parkinson's disease, Acta Neurol. Scand. 119 (2009) 332-337.

[24] N. Vila, J. Castillo, A. Dávalos, A. Esteve, A.M. Planas, Á. Chamorro, Levels of antiinflammatory cytokines and neurological worsening in acute ischemic stroke, Stroke 34 (2003) 671-675.

[25] H.J. Rideout, P. Dietrich, M. Savalle, W.T. Dauer, L. Stefanis, Regulation of alphasynuclein by bFGF in cultured ventral midbrain dopaminergic neurons, $\mathrm{J}$. Neurochem. 84 (2003) 803-813.

[26] C.W. Shults, J. Ray, K. Tsuboi, F.H. Gage, Fibroblast growth factor-2-producing fibroblasts protect the nigrostriatal dopaminergic system from 6-hydroxydopamine, Brain Res. 883 (2000) 192-204.

[27] R. Yilmaz R, A.P. Strafella, A. Bernard, C. Schulte, L. van den Heuvel, N. Schneiderhan-Marra, T. Knorpp, T.O. Joos, F. Leypoldt, J. Geritz, C. Hansen, S. Heinzel, A. Apel, T. Gasser, A.E. Lang, D. Berg, W. Maetzler, C. Marras, Serum inflammatory profile for the discrimination of clinical subtypes in Parkinson's disease, Front. Neurol. 9 (2018) 1123.

[28] C. Lauro, M. Catalano, F. Trettel, C. Limatola, Fractalkine in the nervous system: neuroprotective or neurotoxic molecule? Ann. N. Y. Acad. Sci. 1351 (2015) $141-148$.

[29] A.E. Cardona, E.P. Pioro, M.E. Sasse, V. Kostenko, S.M. Cardona, I.M. Dijkstra, et al. Control of microglial neurotoxicity by the fractalkine receptor, Nat. Neurosci. 9 (2006) 917-924.

[30] J.M. Morganti, K.R. Nash, B.A. Grimmig, S. Ranjit, B. Small, P.C. Bickford, 
C. Gemma, The soluble isoform of CX3CL1 is necessary for neuroprotection in a mouse model of Parkinson's disease, J. Neurosci. 32 (2012) 14592-14601.

[31] M.M. Pabon, A.D. Bachstetter, C.E. Hudson, C. Gemma, P.C. Bickford, CX3CL1 reduces neurotoxicity and microglial activation in a rat model of Parkinson's disease, J. Neuroinflammation 8 (2011) 9.

[32] M. Shi, J. Bradner, A.M. Hancock, K.A. Chung, J.F. Quinn, E.R. Peskind, et al., Cerebrospinal fluid biomarkers for Parkinson disease diagnosis and progression, Ann. Neurol. 69 (2011) 570-580.

[33] M.K. Herbert, M.B. Aerts, M. Beenes M, N. Norgren, R.A. Esselink, B.R. Bloem, et al., CSF neurofilament light chain but not FLT3 ligand discriminates parkinsonian disorders, Front. Neurol. 6 (2015) 91.

[34] E. Silajdžić, R. Constantinescu, B. Holmberg, M. Björkqvist, O. Hansson, Flt3 ligand does not differentiate between Parkinsonian disorders, Mov. Disord. 29 (2014) 1319-1322.

[35] V. Refolo, F. Bez, A. Polissidis, D. Kuzdas-Wood, E. Sturm, M. Kamaratou,

W. Poewe, L. Stefanis, M. Angela Cenci, M. Romero-Ramos, G.K. Wenning,

N. Stefanova, Progressive striatonigral degeneration in a transgenic mouse model of multiple system atrophy: translational implications for interventional therapies,

Acta Neuropathol. Commun. 6 (2018) 2. 